\title{
Robust Artificial Neural Network for Reliability and Sensitivity Analysis of Complex Non-Linear Systems
}

\author{
Uchenna Oparaji ${ }^{\mathrm{a}, \mathrm{b}}$, Rong-Jiun Sheu ${ }^{\mathrm{b}}$, Mark Bankhead ${ }^{\mathrm{c}}$, Jonathan Austin ${ }^{\mathrm{c}}$, \\ Edoardo Patelli ${ }^{\mathrm{a}, *}$ \\ ${ }^{a}$ Institute for Risk and Uncertainty, University of Liverpool, Chadwick Building, Peach \\ Street, Liverpool L69 7ZF, United Kingdom \\ ${ }^{b}$ Institute of Nuclear Engineering and Science, National Tsing Hua University, Hsinchu, \\ Taiwan \\ ${ }^{c}$ National Nuclear Laboratory, Chadwick House, Warrington Rd, Birchwood Park, \\ Warrington, Cheshire, WA3 6AE, United Kingdom
}

\begin{abstract}
Artificial Neural Networks (ANNs) are commonly used in place of expensive models to reduce the computational burden required for uncertainty quantification, reliability and sensitivity analysis. ANN with selected architecture is trained with the back-propagation algorithm from few data representatives of the input/output relationship of the underlying model of interest. However, different performing ANNs might be obtained with the same training data as a result of the random initialization of the weight parameters in each of the network, leading to an uncertainty in selecting the best performing ANN. On the other hand, using cross-validation to select the best performing ANN based on the ANN with the highest $R^{2}$ value can lead to biassing in the prediction. This is as a result of the fact that the use of $R^{2}$ cannot determine if the prediction made by $\mathrm{ANN}$ is biased. Additionally, $R^{2}$ does

\footnotetext{
*Corresponding author

Email addresses: u.oparaji@liverpool.ac.uk (Uchenna Oparaji),
} epatelli@liverpool.ac.uk (Edoardo Patelli)


not indicate if a model is adequate, as it is possible to have a low $R^{2}$ for a good model and a high $R^{2}$ for a bad model. Hence in this paper, we propose an approach to improve the robustness of a prediction made by ANN. The approach is based on a systematic combination of identical trained ANNs, by coupling the Bayesian framework and model averaging. Additionally, the uncertainties of the robust prediction derived from the approach are quantified in terms of confidence intervals. To demonstrate the applicability of the proposed approach, two synthetic numerical examples are presented. Finally, the proposed approach is used to perform a reliability and sensitivity analysis on a process simulation model of a UK nuclear effluent treatment plant developed by National Nuclear Laboratory (NNL) and treated in this study as a black-box employing a set of training data as a test case. This model has been extensively validated against plant and experimental data and used to support the UK effluent discharge strategy.

Keywords:

Monte-Carlo Simulation, Global Sensitivity Analysis, Reliability Analysis, Artificial Neural Network, Uncertainty Quantification

\section{Introduction}

Complex critical systems, such as bridges, buildings, nuclear plants, and air-crafts, are designed to fulfil specific performance requirements despite the unavoidable uncertainty. Therefore, their respective designs should be able to deal with changing conditions driven by nature. Due to the infeasibility (i.e. huge cost, time) in testing the performance of these systems for varying levels of uncertainties, mathematical models and virtual prototypes are used 
in simulating the behaviour of these systems. This advance in computational development has allowed engineering practitioners to reduce the number of expensive test required to qualify a new system/product. On the other hand, a quantifiable mathematical model simulating the performance of a system is viewed to be composed of three main elements such as: 1) an input vector that represents the state variables of the system, 2) a mathematical model defining the system of interest, which is usually seen as a black-box, and finally 3) an output vector that represents the performance of the system. Two types of uncertainties that affects the state variables of the mathematical model are usually considered: 1) randomness due to inherent variability in the system behaviour (aleatory uncertainty) and, 2) imprecision due to lack of knowledge and information on the system (epistemic uncertainty). Usually, the design of complex critical systems requires the explicit consideration of the different levels of uncertainties affecting the state variables of the system for an adequate performance assessment $[1,2]$.

\subsection{Reliability and Sensitivity Analysis for Complex System Performance Evaluation}

To quantify the performance of complex critical systems in the presense of uncertainties, reliability analysis is usally carried out. In mathematical terms, the state variables of a system is defined by a vector collection $\mathbf{X}=$ $\left(X_{1}, X_{2}, \ldots, X_{p}\right)$ of state variables. The performance criteria of the system (i.e. limit state) $g(X)$ divides the system state into two regions (i.e. safe domain $S=X: g(X)>0$ and a failure domain $F=X: g(X) \leq 0)$. The probability that the system would not meet an expected performance using Monte Carlo method can be expressed as $\hat{p_{F}}=1 / N \sum_{i}^{N} I\left(g\left(X_{i}\right)\right)$, where the 
indicator function $I\left(g\left(X_{i}\right)\right)$ is 1 if $g\left(X_{i}\right)$ is negative or 0 otherwise. It should be noted that among the numerical methods proposed in several literature to estimate $\hat{p_{F}}$, simulation methods [3] have attracted significant attention due to their flexibility and accuracy. Simulation methods are generally applicable to varying systems, but require a balance between computational efficiency and accuracy. These simulation methods includes: Monte Carlo (MC) [4], Importance Sampling [5], Directional Sampling [6], Line Sampling [7, 8], Subset Simulation $[9,10]$ etc. Each of these simulation methods have special features to target different classes of problems. For instance, in a scenario when the target failure probability $\hat{p_{F}}$ is less than $10^{-4}$, direct Monte Carlo method [4] is not suited for this problem. Hence, a simulation approach that is suitable for the problem can be adopted (i.e. Subset simulation, Line sampling). Similarly, a system performance can only be improved if the state variables that affect the performance significantly are identified and focused on. Sensitivity analysis is used to achieve this by identifying and ranking the contributions of each state variable of the system to the variability in the performance. Most often, the variance based method to sensitivity analysis [11] is adopted when assessing the contributions of the state variables. This method is a class of simulation approaches that is used to decomposes the output variance into parts that can be attributed to the inputs and interactions between them. The sensitivity indices (i.e. state variable ranking) using this approach are estimated by $S_{i}=V_{i} / \operatorname{Var}(Y)$ and $T_{i}=1-\operatorname{Var}_{X \sim i}\left[E_{X \sim i}\left(Y \mid X_{\sim i}\right)\right] / \operatorname{Var}(Y)$, where $S_{i}$ is the contribution of a single state variable and $T_{i}$ is the contribution due to interactions among the state variables. 


\subsection{Need for Surrogate Models}

Unfortunately, reliability and sensitivity analysis using the simulation approach are computationally demanding tasks, requiring a huge number of model runs. To be specific, when performing reliability analysis on a high reliable system with a low failure probability (i.e. $p_{F}<10^{-4}$ ), a huge number of samples $N$ is required to accurately compute the failure probability (i.e. $\left.N>1 / p_{F}\right)$. Similarly, when performing sensitivity analysis on a model with $p$ number of state variables using the variance based method [12], the total number of model evaluations $E_{M}$ follows the relationship $E_{M}=(p+$ 2) $N$, where $N$ is the number of samples required. The number of samples $N$ is usually proportional to the dimension of the model being analysed. For instance, Patelli et al., (2012) [13] required greater than $10^{5}$ samples to accurately compute the sensitivity indices of the Gravity Field and SteadyState Ocean Circulation Explorer (GOCE) satellite due to the complexity of the finite element (FE) model used (i.e. $p>3000$ )(see ref [14] also). In a similar fashion, Baroni and Tranola (2014) [15] found out that the sensitivity indices convergence was reached using $N=1024$ samples for a model of $p=5$ uncertain parameters. Generally speaking, the computational cost required for performing the aforementioned analysis can vary amongst different set of models. This is as a result of the time required for a single run of the model. Therefore, to tackle these huge computation restrictions, alternative methods that significantly reduces this computational burden must be sourced out.

\subsection{Artificial Neural Networks for Reliability and Sensitivity Analysis}

Artificial Neural Network (ANN) is a computing device inspired by the

neurology of the brain [16]. Over the past few decades, ANN has proven to be 
an extremely valuable tool for reducing the computational burden required for performing reliability and sensitivity analysis. For instance, in refs [13, 17-20] ANNs have been used as substitutes to replace expensive models in order to speed up their analysis. The ANNs in the aforementioned literature have been trained with the back-propagation algorithm [21] based on inputoutput training data set $D_{\text {train }}(x, y)$ extracted from the expensive model of interest. The back-propagation algorithm efficiently computes the slope of the gradient by employing a gradient descent, following the slope of the error function downward along, and simultaneously changing all the weight values of the network. The weight parameters are constantly tuned until the value no longer decreases. However, a limitation of using the back-propagation algorithm to train an ANN is that different performing ANNs (i.e. with identical architecture) arise from the same training data. This is as a result of the gradient decent algorithm used to minimize the error function of an ANN, getting trapped on a different error surface in each ANN. Consequently, cross-validation is utilized to select the best performing ANN in the set by choosing the ANN with the lowest validation regression error (i.e. highest $R^{2}$ ). However, a key limitation of using $R^{2}$ to judge the performance of ANN is that it cannot determine whether the weight parameters and predictions of the ANN are biased, as it is possible to have a low $R^{2}$ value for a good ANN, and a high $R^{2}$ value for an ANN that does not fit the data. Moreover, if the validation data is partially corrupted with noise, the evaluation of the $R^{2}$ will be biased. Hence, we make a claim (see Section 3.2 for verification of claim) that the use of $R^{2}$ value to select the best performing ANN in a set of different performing ANNs will introduce biassing in the quantity 
predicted by the selected ANN. Therefore, in this paper, we aim to address this issue by proposing a simple novel approach used to reduce the biasing and improve the robustness of the prediction made by an ANN. The structure of this paper is organized as follows: In Section 2, the proposed approach is discussed. Next, two synthetic numerical examples are presented in Section 3 to demonstrate the applicability of the proposed approach. This is followed by applying the approach to a real case study involving a radioactive waste treatment plant. Finally, conclusions are provided in Section 5.

\section{Proposed Approach}

The proposed approach in this paper is aimed towards improving the robustness of the prediction made by an ANN when used to perform reliability and sensitivity analysis. The underlying principle behind the proposed approach is to construct a set of ANNs (i.e. same architecture) based on the same training data $D_{\text {train }}(x, y)$. By doing so, a distribution of similar ANNs whose error functions are trapped in different local minima is created. The major highlight of this approach is that the solution space of the error function is exploited as many times as possible with the possibility of locating a global minima on the error surface. Further, Bayes' theorem is used to evaluate the posterior probability of each of the trained ANN based on their likelihood to predict the training data. This is followed by the use of a model averaging technique (adjustment factor approach see [22]) to combine the total prediction made by all the ANNs in the set to yield a robust prediction that converges to the true value. Finally, the model uncertainty propagated to the predicted quantity is quantified in terms of confidence intervals. 


\subsection{Bayesian Model Selection for Identical Trained Artificial Neural Net-}

works

Various authors (see e.g.[23, 24]) have used Bayesian model selection (BMS) technique as a means of selecting an appropriate model structure for their respective problems, by computing the model evidence based on Markov Chain Monte Carlo (MCMC) posterior simulations of the model parameters or using approximation techniques to estimate the posterior probability of a particular model. Hence, BMS techniques have been used in place of the standard optimization training technique (e.g. back-propagation) to identify the "best" model.

Conversely, the aim of this paper is different. The optimal network architecture of the ANN is assumed to be known (e.g. determined by heuristic approach and trained by the back-propagation algorithm). Then, the optimal ANN is trained multiple times and a set of different performing networks is obtained. BMS have been used to select and rank the identical trained networks. By so doing, the posterior probability of a network in the identical set can be defined as the degree of belief that its given prediction is true, given that one of the identical trained network in the set has its error function located in the global minima. However, from a practical point of view all the networks in the set are just approximations of a high fidelity model. For this reason, it is more appropriate to interpret the posterior probability as the degree of belief that a particular ANN within the set is the best approximation of the underlying model of interest. Therefore, given a set of $M$ identical competing ANNs $\left(N_{1}, N_{2}, \ldots, N_{M}\right)$ trained with same data $D_{\text {train }}(x, y)$, the posterior probability of the $k^{\text {th }}$ ANN, i.e. the $N_{k}$ in the set, is defined by 
Eq.(1):

$$
P\left(N_{k} \mid D_{\text {train }}\right)=\frac{P\left(D_{\text {train }}(x, y) \mid N_{k}\right) P\left(N_{k}\right)}{\sum_{q=1}^{M} P\left(D_{\text {train }}(x, y) \mid N_{q}\right) P\left(N_{q}\right)}
$$

where $P\left(D_{\text {train }}(x, y) \mid N_{k}\right)$ is the likelihood of training data $D_{\text {train }}(x, y)$ for the $N_{k} \mathrm{ANN}$, and $P\left(N_{k}\right)$ is the prior probability of $N_{k}$, which is the ANN probability evaluated before observing training data $D_{\text {train }}(x, y)$. The prior ANN probability $P\left(N_{k}\right)$ can be specified depending on the existing prior knowledge about the credibility of $\mathrm{ANN} N_{k}$, or it can be given as a uniform probability, $P\left(N_{k}\right)=1 / M$, if no additional information is provided. The advantage of assigning uniform prior probability to $P\left(N_{k}\right)$ is that the difficulty of estimating the prior probability numerically is avoided. The likelihood $P\left(D_{\text {train }}(x, y) \mid N_{k}\right)$ may be thought of as the probability of observing the training data $D_{\text {train }}(x, y)$ under ANN $N_{k}$. It supplies a relative measure of how well the ANN $N_{k}$ is supported by the training data $D_{\text {train }}(x, y)$. Since the denominator in Eq.(1) is common for all the ANNs, the posterior ANN probability is proportional to prior probability and the likelihood. The likelihood of each $\mathrm{ANN}$ is evaluated by measuring the degree of agreement between the training data $D_{\text {train }}(y)$ and the response $\hat{y}$ for each ANN. Hence, a probabilistic relationship between training data $D_{\text {train }}(x, y)$ and ANN predictions $\hat{y}$ involving uncertainty can be described. Typically, the bias function and noise are included as parts of the probabilistic relationship to match ANN predictions with training data. The bias function captures the discrepancies between the expensive model responses and predictions made by the ANN. The noise is usually assumed to be independent and identically distributed normal random variable with a mean of zero [25]. Various authors [26-28] have used the Bayesian statistical methodology to quantify the uncertainty 
in the bias function modelled as a Gaussian process. In their works, a mathematical formulation that combines bias function associated with the ANN and noise from training data is utilized to describe the probabilistic relationship between the training data $D_{\text {train }}(x, y)$ and ANN predictions $\hat{y}$. The mathematical formulation of this probabilistic relationship is given by the following equation:

$$
D_{\text {train }}(y)=\hat{y}-\varepsilon
$$

where $\varepsilon$ is a random variable that covers both bias associated with the ANN prediction $\hat{y}$ and the noise in the response training data $D_{\text {train }}(y) . \varepsilon$ is assumed to be an independent identically distributed random variable with a mean $\mu$ of zero. The use of $\varepsilon$ with zero mean does not shift ANN prediction $\hat{y}$. This reflects the fact that $\hat{y}$ is the most probable prediction value for the ANN. The bias function is not included as a separate term in the probabilistic relationship. This is due to the fact that introducing a separate bias function results in shifting the prediction $\hat{y}$ of the ANN from the initially predicted value. The likelihood $P\left(D_{\text {train }}(x, y) \mid N_{k}\right)$ of training data $D_{\text {train }}(x, y)$ for ANN $N_{k}$ is evaluated by observing where the training data points $D_{\text {train }}(y)$ are located in the distribution of $\hat{y}$ estimated by $N_{k}$. The procedures to estimate the distribution $P\left(\hat{y} \mid N_{k}\right)$ of $N_{k}$ and the likelihood $P\left(D_{\text {train }}(x, y) \mid N_{k}\right)$ is given. First, the uncertainty in errors of predictions $\hat{y}$ made by $N_{k}$ is quantified by introducing an assumption that the prediction errors are independent and identically distributed normal random variable with a mean $\mu$ of zero. The error of the prediction of the $k^{\text {th }}$ network is represented by the following:

$$
\varepsilon_{k i}=D_{\text {train }}\left(y_{i}\right)-\hat{y}_{i}, \varepsilon_{k i} \sim N\left(0, \sigma_{k}^{2}\right), i=1,2, \ldots, N
$$


where $D_{\text {train }}\left(y_{i}\right)$ is the $i^{\text {th }}$ training response output data, $\hat{y}_{i}$ the prediction of the training data made by $N_{k}, \sigma_{k}^{2}$ is the variance of prediction error $\varepsilon_{k i}$, and $N$ the number of samples in the training data. The prediction error $\varepsilon_{k i}$ measured is considered to be a random sample from a normal distribution with a mean $(\mu)$ of zero and variance $\sigma_{k}^{2}$. Using the principle of maximum likelihood estimation (MLE) (see [29]), the variance $\sigma_{k}^{2}$ for $N_{k}$ can be estimated as:

$$
\sigma_{k}^{2}=\frac{1}{N} \sum_{i=1}^{N} \varepsilon_{k i}^{2}
$$

Secondly, the predictive distribution $P\left(\hat{y} \mid N_{k}\right)$ of response $\hat{y}$ under model $N_{k}$ is created by including the prediction error obtained in the previous step into the prediction of $\hat{y}$ made by $N_{k}$. This predictive distribution is defined by the following equation:

$$
P\left(\hat{y} \mid N_{k}\right)=D_{\text {train }}(y)+\varepsilon_{k i}
$$

Lastly, assuming that the residuals between the training data $D_{\text {train }}(x, y)$ and $N_{k}$ output $\hat{y}$ are normally and independently distributed with a mean of zero and constant variance $\sigma_{k}^{2}$, the likelihood function $P\left(D_{\text {train }}(x, y) \mid N_{k}\right)$ is approximated by:

$$
P\left(D_{\text {train }}(x, y) \mid N_{k}\right) \approx \frac{1}{\sqrt{2 \pi \sigma_{k}^{2}}} \frac{1}{N} \sum_{i=1}^{N} \exp \left\{\frac{-\left[y_{i}-\hat{y}_{k i}\right]^{2}}{2 \sigma_{k}^{2}}\right\}
$$

\subsection{Robust Artificial Neural Network Prediction}

To obtain a robust prediction from an ANN, the estimates made by all the subsequent trained ANNs are combined using model averaging technique. 
Specifically, the adjustment factor approach (see [22]) which is a model averaging technique is combined with Bayes' theorem. With this approach, the ANN having the highest posterior probability is used in conjunction with other respective ANNs trained to correct the bias estimate predicted by a single ANN. The adjustment factor is evaluated by assuming the error between the prediction of all the subsequent trained ANNs and the training data are normally distributed. For the quantification of the robust value, the posterior probability computed for each ANN is used as a weighting. A distribution from the response predicted by the ANNs is created by introducing the adjustment factor $A_{f}$ which is characterized by a normal distribution. The robust ANN prediction can be obtained from the following equation:

$$
y_{\text {robust }}=\hat{y}^{*}+A_{f}
$$

where $\hat{y}^{*}$ represents the point estimate of the best ANN in the set with the highest probability, $A_{f}$ represents the adjustment factor, and $y_{\text {robust }}$ represent the robust prediction which also incorporates the model uncertainty. Since the adjustment factor $A_{f}$ is assumed to be a normal distribution, the expected value and variance of the adjustment factor $A_{f}$ is given by the following relationships:

$$
\begin{gathered}
E\left(A_{f}\right)=\sum_{k=1}^{M} P\left(N_{k} \mid D_{\text {train }}\right)\left(\hat{y}_{k}-\hat{y}^{*}\right) \\
\operatorname{Var}\left(A_{f}\right)=\sum_{k=1}^{M} P\left(N_{k} \mid D_{\text {train }}\right)\left(\hat{y}_{k}-E\left(y_{\text {robust }}\right)\right)^{2}
\end{gathered}
$$

Similarly, the expected value and variance of the robust prediction $y_{\text {robust }}$ can be estimated from the following relationships:

$$
E\left(y_{\text {robust }}\right)=\hat{y}^{*}+E\left(A_{f}\right)
$$




$$
\operatorname{Var}\left(y_{\text {robust }}\right)=\operatorname{Var}\left(A_{f}\right)
$$

where $E\left(A_{f}\right)$ and $\operatorname{Var}\left(A_{f}\right)$ represents the expected value and variance of the adjustment factor, and $E\left(y_{\text {robust }}\right)$ and $\operatorname{Var}\left(y_{\text {robust }}\right)$ represents the expected value and variance of the robust estimate.

\subsection{Confidence Interval for Robust Estimate}

To quantify the uncertainty in the robust prediction $y_{\text {robust }}$ due to model uncertainty, confidence intervals are established. In particular, $5^{\text {th }}$ and $95^{\text {th }}$ percentiles derived from the robust prediction are used quantify the model uncertainty. In theory, this interval is likely to contain the true estimated value. As the model uncertainty is assumed to follow normal distribution, the confidence intervals (see [30]) are calculated from the following equations:

$$
\begin{aligned}
& \overline{C I}=E\left(y_{\text {robust }}\right)+1.96 \sqrt{\operatorname{Var}\left(y_{\text {robust }}\right)} \\
& \underline{C I}=E\left(y_{\text {robust }}\right)-1.96 \sqrt{\operatorname{Var}\left(y_{\text {robust }}\right)}
\end{aligned}
$$

where $\overline{C I}$ and $\underline{C I}$ represents the upper and lower confidence intervals of the robust estimate.

\section{Numerical Examples}

To demonstrate the applicability of the approach presented, two synthetic numerical examples are used for illustrative purpose. 


\subsection{Example 1}

The first example presents a 2-D non-linear function. The output of the function $Y$ is represented by the equation:

$$
Y=10 \cos (X 1)+10 \sin (X 2)
$$

where the model state variables $X 1$ and $X 2$ are uniformly and independently distributed in the range of 0 and 360 degrees. For this current reliability analysis, the failure criteria $(F=g(X) \leq 0)$ of the model is defined as when the model output $Y$ exceeds 15 . The surface and contour plot of the function is shown in Fig.1 and 2.

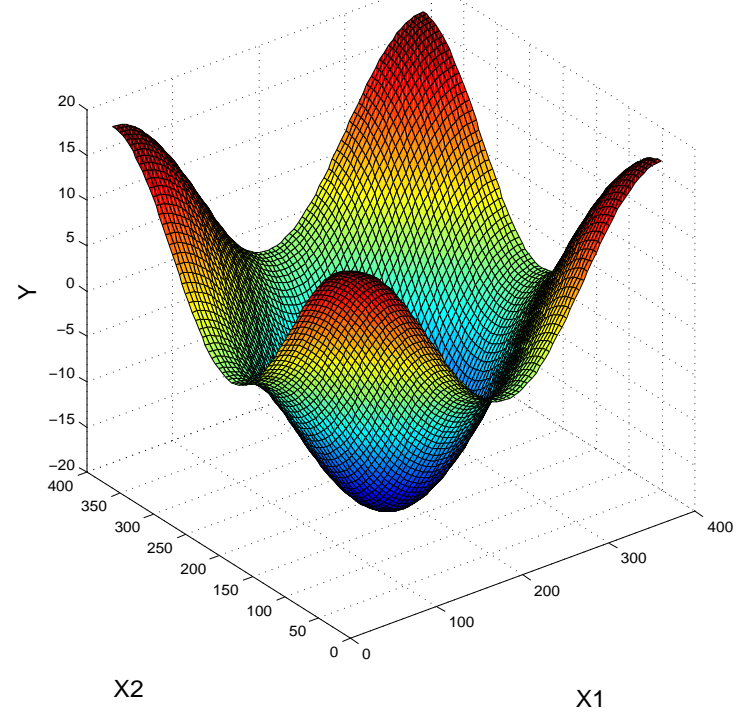

Figure 1: Surface Plot of Safety Function 


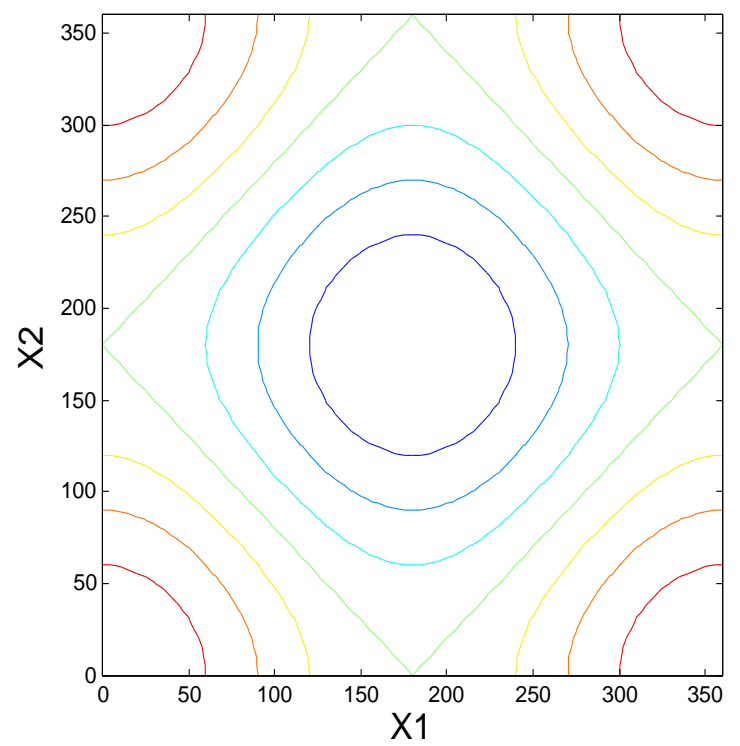

Figure 2: Contour Plot of Safety Function

Given that the proposed will be tested, the objectives of this example will involve training multiple ANNs based on the same architecture, compute the failure probability $\hat{p_{F}}$ from each of the each of the ANNs based on the failure criteria $(F=g(X) \leq 0)$ defined, compute the robust estimate of $\hat{\left(p_{F}\right)}$, and finally quantify the uncertainty in the the robust estimate of $\left.\hat{(} p_{F}\right)$ due to model uncertainty.

\subsection{Analysis}

Training samples $D_{\text {train }}(x, y)$ of size $N=100$ have been generated via Latin hypercube sampling (LHS) algorithm[31] from Eq.(14). Two sets $Z_{1}=$ $N_{i}, i=1,2, \ldots M$ and $Z_{2}=N_{i}, i=1,2, \ldots M$ composed of $M=10$ identical ANNs have been trained based on $D_{\text {train }}(x, y)$. Specifically, in the first set $\left(Z_{1}\right)$, all the training samples in $D_{\text {train }}(x, y)$ have been used to train the ANNs to maximize their predictive performances. For the second set $Z_{2}, 80 \%$ of the 
training samples $D_{\text {train }}$ have been used to train the ANNs and the remaining $20 \%$ used for validation. The network architecture chosen for the ANNs in both sets composed of three hidden layers $(2,5,1)$. Next, the posterior probability of the ANNs in set $Z_{1}$ has been estimated using Bayes' formula given in Eq. (1) by assigning uniform prior probability $P\left(N_{k}\right)=1 / M$ to each ANN. On the other hand, the $R^{2}$ validation values for the ANNs in set $Z_{2}$ have been estimated based on the validation samples. The results of these analysis are given in Table. 1 for robust comparison. It should be noted that $i^{\text {th }}$ ANN in both set $\left(Z_{1}\right.$ and $\left.Z_{2}\right)$ have been trained inside the same iteration loop, hence it is assumed that their resultant behaviour should be similar, except that the ANNs in $Z_{1}$ are expected to have a higher performance capability due to more training samples used to train them. As shown in Table.1, although the ANNs $N_{i}, i=1,2, . . M$ in sets $Z_{1}$ and $Z_{2}$ are identical as they have been trained in the same iteration loop, there is no agreement between the posterior probability estimated from the ANNs in $Z_{1}$ and the corresponding $R^{2}$ validation values estimated from the ANNs in $Z_{2}$. This finding is further supported by the fact that the best model selected $\left(N_{1}\right)$ based on its posterior probability has the lowest $R^{2}$ value. Hence, we can support our claim that the use of $R^{2}$ value to select the best model is a biased method. Further, to implement the proposed approach, the ANNs in $Z_{1}$ have been chosen as they have higher predictive capability (i.e. more samples used to train them). To accurately compute a robust estimate of $\hat{p_{F}}, 10^{4}$ Monte Carlo simulation runs have been used for each ANN, and the proposed approach presented have been used to average out the prediction made by each ANN model into a robust value that is converges to the true 
value. Finally, the model uncertainty propagated to robust prediction of $\hat{p_{F}}$ has been quantified in terms of confidence intervals estimated from Eq.(12) and Eq.(13).

Table 1: Simulation Results for Non-linear Function

\begin{tabular}{|l|l|l|l|l|}
\hline$A N N$ & $P\left(N_{k} \mid D\right)\left(Z_{1}\right)$ & $R^{2}\left(Z_{2}\right)$ & $\hat{p_{F}}\left(Z_{1}\right)$ & $\sigma_{k}^{2}\left(Z_{1}\right)$ \\
\hline 1 & $9.8 \mathrm{E}-1$ & 0.9850 & 0.080 & 0.0430 \\
\hline 2 & $1.6 \mathrm{E}-3$ & 0.9993 & 0.088 & 0.0553 \\
\hline 3 & $7.1 \mathrm{E}-3$ & 0.9993 & 0.078 & 0.0547 \\
\hline 4 & $8.7 \mathrm{E}-3$ & 0.9929 & 0.086 & 0.6188 \\
\hline 5 & $2.8 \mathrm{E}-6$ & 0.9992 & 0.087 & 0.0603 \\
\hline 6 & $7.0 \mathrm{E}-4$ & 0.9993 & 0.076 & 0.0583 \\
\hline 7 & $4.0 \mathrm{E}-4$ & 0.9992 & 0.090 & 0.0597 \\
\hline 8 & $3.0 \mathrm{E}-3$ & 0.9993 & 0.105 & 0.0532 \\
\hline 9 & $6.4 \mathrm{E}-8$ & 0.9988 & 0.081 & 0.0859 \\
\hline 10 & $6.9 \mathrm{E}-8$ & 0.9988 & 0.080 & 0.0913 \\
\hline
\end{tabular}




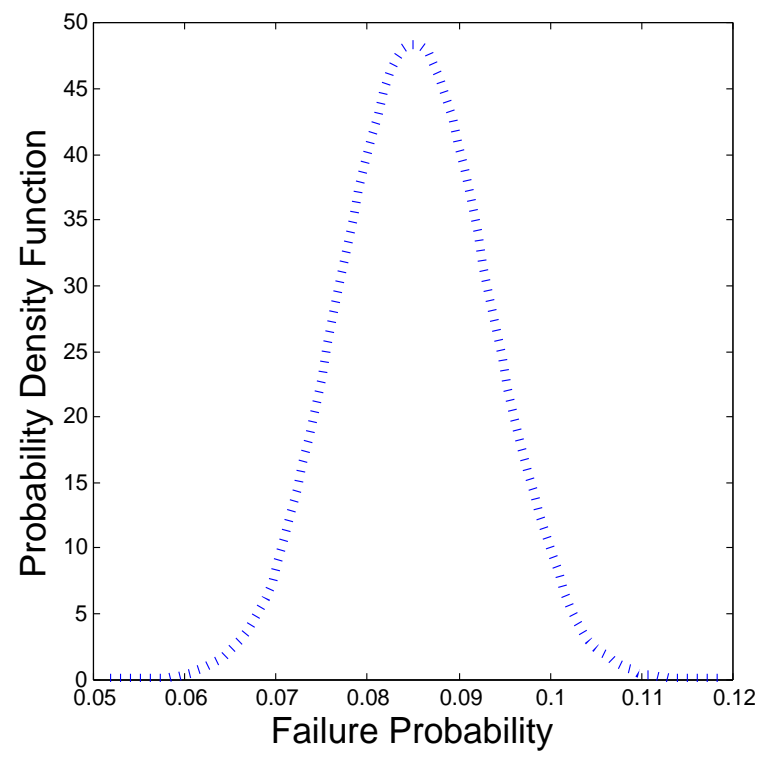

Figure 3: Model Uncertainty Propagated to $\hat{p_{F}}$ Using the Proposed Approach

\subsection{Verification of the Proposed Approach for Reliability Analysis}

To verify that the true value of $\hat{p_{F}}$ falls within the robust confidence interval derived from the approach, the real model given in Eq.(14) has been used to estimate the true value of $\hat{p_{F}}$ adopting the same failure criteria (i.e. $Y>15$ ). Similarly, $10^{4}$ Monte Carlo simulation runs have been used to estimate the true value of the failure probability $\hat{p_{F}}=0.0870$. This value obtained $\left(\hat{p_{F}}=0.0870\right)$ verifies that the proposed approach is robust enough to estimate a prediction that converges to the true value. Finally, to investigate the number of ANNs that must be trained for the prediction (i.e. $\hat{p}_{F}$ ) that converges to the true unbiased value, a different number ( $M=10,100,1000,10000)$ of ANNs have been trained repeatedly adopting the proposed approach. For each set of $(M=10,100,1000,10000)$ ANNs trained, the confidence intervals $\overline{C I}, \underline{C I}$ and expected value of the robust 
prediction $E\left(y_{a d j}\right)$ has been estimated. The results are shown in Fig.(4).

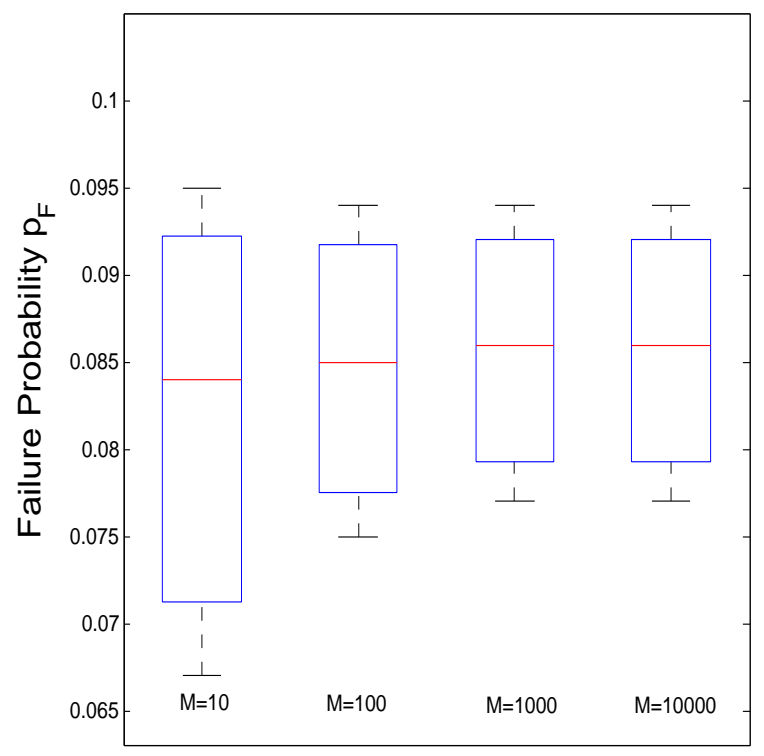

Figure 4: Confidence Intervals of $p_{F}$ for Different Number of Trained ANNs

From the results shown in Fig.4, it is evident that as the number of ANNs trained increases, the robust prediction obtained from the approach converges to the true value. The reason for this outcome is that as more identical ANNs are being trained, the error function solution space is explored many times as possible, hence, the global minimum of the error function is more likely to be reached during this exploration. Thus, the value estimated from the proposed approach is likely to approach the true value. On the other hand, although the computational cost required to achieve the best solution of the predicted quantity using the approach is computationally expensive, parallelization strategies could be adopted and incorporated into the analysis to improve the computational efficiency of the approach. 


\subsection{Example 2}

The second example used to test the proposed approach adopts the well known the Ishigami function. This function is often used as a benchmark model to test different sensitivity analysis methods. The function is represented by the following equation:

$$
f(X)=\sin (X 1)+a \sin ^{2} X 2+b X 3^{4} \sin (X 1)
$$

In this example, the numerical values chosen for $a$ and $b$ are 7 and 0.1 respectively. The parameters $X i, i=1,2,3$ are uniformly distributed in the interval of $-\pi$ and $\pi$. The surface plot of the non-linear function is shown in Fig.5.

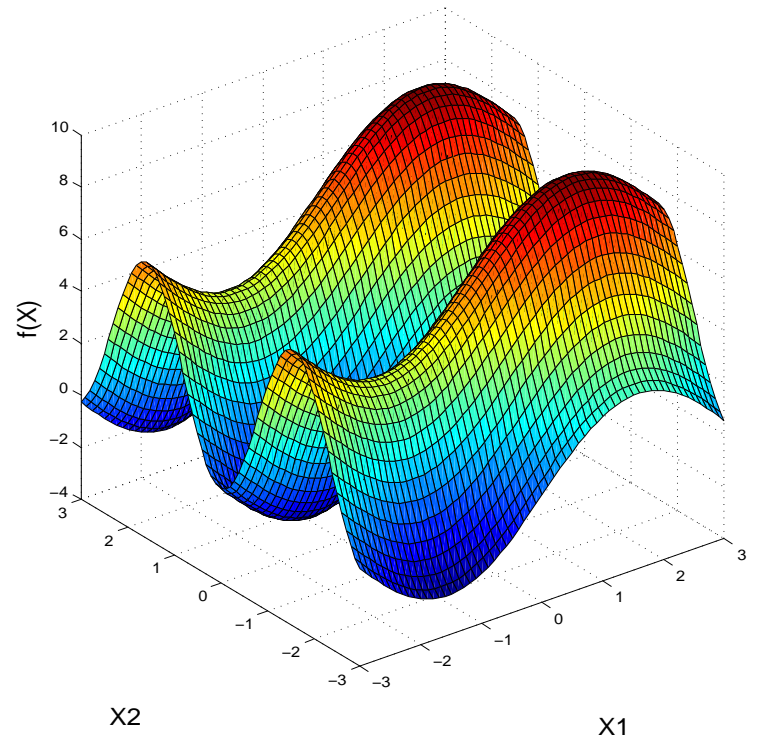

Figure 5: Ishighami fuction: Relationship between X1 and X2

The overall objective of this example is to adopt the proposed approach to increase the robustness of the sensitivity indices predicted by ANN and quantify the ANN model uncertainties. 


\subsection{Analysis}

Here, training samples $D_{\text {train }}$ of size $N=100$ have been generated via Latin hypercube algorithm [31]. Once again, two sets $Z_{1}=N_{i}, i=1,2, \ldots M$ and $Z_{2}=N_{i}, i=1,2, \ldots M$ containing $M=10$ ANNs with a three hidden layer configuration of $(3,13,1)$ have been constructed and used as a substitute for the Ishigami function. In a similar fashion as carried out in the first example, the ANNs in the first set $\left(Z_{1}\right)$ have been trained with $100 \%$ of $D_{\text {train }}(x, y)$, while $80 \% D_{\text {train }}(x, y)$ have been used to train the ANNs in the second set $\left(Z_{2}\right)$, and the remaining $20 \%$ used to compute the $R^{2}$ validation error. Using Bayes' formula given in Eq. (1) the posterior probability of each ANN in $Z_{1}$ have been estimated by assuming a uniform prior probability $P\left(N_{k}\right)=1 / M$. Tables.2 and 3 shows the summary of the results obtained. Again, comparing the posterior probability and the $R^{2}$ validation error shows that there is no correlation between them. For instance, the $9^{\text {th }}$ and $10^{\text {th }}$ ANN have the highest $R^{2}$ values, however, their respective posterior probability are relatively low compared to other ANNs. Hence, selecting them based on their $R^{2}$ validation error can result to biased values, when used for prediction. Henceforth, the ANNs in $Z_{1}$ have been selected to implement the proposed approach. To compute the robust predicted quantity, Saltelli's algorithm [32] has been used to estimate $S_{i}$ and $T_{i}$ for all the ANNs adopting $N=10^{5}$ Monte Carlo samples. Further, the proposed approach has been used to combine the sensitivity indices estimated to give a robust estimate. Finally, the model uncertainties propagated to the robust estimates of the sensitivity indices are quantified in terms of confidence intervals (see Tables. 4 and 5). 
Table 2: Quantities Estimated from Identical ANNs (NB: $X_{i}, i=1,2,3$ is the First Order Sensitivity Indices Estimated from $Z_{1}$ )

\begin{tabular}{|l|l|l|l|l|l|}
\hline$A N N$ & $R^{2}\left(Z_{2}\right)$ & $\sigma_{k}^{2}\left(Z_{1}\right)$ & $X 1\left(Z_{1}\right)$ & $X 2\left(Z_{1}\right)$ & $X 3\left(Z_{1}\right)$ \\
\hline 1 & 0.9549 & 0.5382 & 0.3266 & 0.4647 & 0.0014 \\
\hline 2 & 0.9546 & 0.5462 & 0.3253 & 0.4673 & 0.0009 \\
\hline 3 & 0.9548 & 0.4826 & 0.3210 & 0.4618 & 0.0006 \\
\hline 4 & 0.9604 & 0.4405 & 0.3276 & 0.4681 & 0.0000 \\
\hline 5 & 0.9572 & 0.4983 & 0.3167 & 0.4672 & 0.0054 \\
\hline 6 & 0.9623 & 0.4404 & 0.3204 & 0.4552 & 0.0053 \\
\hline 7 & 0.9567 & 0.4842 & 0.3299 & 0.4657 & 0.0008 \\
\hline 8 & 0.9552 & 0.5059 & 0.3305 & 0.4611 & 0.0007 \\
\hline 9 & 0.9754 & 0.2225 & 0.3130 & 0.4407 & 0.0025 \\
\hline 10 & 0.9741 & 0.5537 & 0.3352 & 0.4763 & 0.0075 \\
\hline
\end{tabular}


Table 3: Quantities Estimated from Identical ANNs (NB: $X_{i}, i=1,2,3$ is the Total Effect Sensitivity Indices Estimated from $Z_{1}$ )

\begin{tabular}{|l|l|l|l|l|}
\hline$A N N$ & $P\left(N_{k} \mid D\right)\left(Z_{1}\right)$ & $X 1\left(Z_{1}\right)$ & $X 2\left(Z_{1}\right)$ & $X 3\left(Z_{1}\right)$ \\
\hline 1 & $6.6 \mathrm{E}-7$ & 0.5338 & 0.4635 & 0.2105 \\
\hline 2 & $3.3 \mathrm{E}-7$ & 0.5295 & 0.4575 & 0.2074 \\
\hline 3 & $1.5 \mathrm{E}-4$ & 0.5357 & 0.4588 & 0.2059 \\
\hline 4 & $1.5 \mathrm{E}-2$ & 0.5338 & 0.4657 & 0.2140 \\
\hline 5 & $3.1 \mathrm{E}-6$ & 0.5403 & 0.4697 & 0.2090 \\
\hline 6 & $1.5 \mathrm{E}-2$ & 0.5230 & 0.4521 & 0.1986 \\
\hline 7 & $1.3 \mathrm{E}-4$ & 0.5393 & 0.4642 & 0.2161 \\
\hline 8 & $1.5 \mathrm{E}-5$ & 0.5343 & 0.4602 & 0.2063 \\
\hline 9 & $1.2 \mathrm{E}-4$ & 0.5428 & 0.4387 & 0.2356 \\
\hline 10 & $1.6 \mathrm{E}-5$ & 0.5392 & 0.4730 & 0.2180 \\
\hline
\end{tabular}

Table 4: Quantified Uncertainty in Robust Pridiction for First Order Sensitivity Indices

\begin{tabular}{|l|l|l|l|l|}
\hline Parameter & $\underline{C I}$ & $\overline{C I}$ & $\operatorname{Var}\left(S_{i}\right)$ & $E\left(S_{i}\right)$ \\
\hline$X 1$ & 0.3138 & 0.3347 & $7.9 \mathrm{E}-6$ & 0.3139 \\
\hline$X 2$ & 0.4416 & 0.4766 & $18.3 \mathrm{E}-6$ & 0.4418 \\
\hline$X 3$ & 0.0029 & 0.0070 & $5.1 \mathrm{E}-6$ & 0.0030 \\
\hline
\end{tabular}


Table 5: Quantified Uncertainty in Robust Pridiction for Total Effect Sensitivity Indices

\begin{tabular}{|l|l|l|l|l|}
\hline Parameter & $\underline{C I}$ & $\overline{C I}$ & $\operatorname{Var}\left(S_{i}\right)$ & $E\left(S_{i}\right)$ \\
\hline$X 1$ & 0.5429 & 0.5462 & $27 \mathrm{E}-6$ & 0.5430 \\
\hline$X 2$ & 0.4388 & 0.4787 & $39 \mathrm{E}-6$ & 0.4389 \\
\hline$X 3$ & 0.2357 & 0.2365 & $40 \mathrm{E}-6$ & 0.2358 \\
\hline
\end{tabular}

\subsection{Verification of Proposed Approach for Sensitivity Analysis}

To verify the robust sensitivity indices obtained from the approach converges to the true value, a comparison has been made with the predicted value estimated from the real model. Adopting $N=10^{5}$ Monte Carlo samples, the real sensitivity indices have been computed directly from the real model with Saltelli's algorithm [32] (see Table.6) The results show that the robust values obtained from the approach is close to the real value estimated from the real model. Hence, we conclude that our approach is sufficient enough to increase the robustness of the prediction made by ANN.

Table 6: Sensitivity Indices Estimated from the Real Model

\begin{tabular}{|l|l|l|}
\hline Parameter & First Order Indices & Total Effect Indices \\
\hline X1 & 0.3139 & 0.5430 \\
\hline X2 & 0.4417 & 0.4389 \\
\hline X3 & 0.0030 & 0.2358 \\
\hline
\end{tabular}




\section{Case Study}

Once again, the applicability of the proposed approach is demostrated by performing reliability and sensitivity analysis on a real case study. This case study focuses on a complex and expensive mathematical model of the Site Ion eXchange Effluent Plant (SIXEP)(see [33]) situated on the nuclear fuel reprocessing and decommissioning site at Sellafield, U.K. Sellafield site is one of the largest nuclear installations in the world and is arguably the most complex nuclear site in the world due to the fact there is a lot of engineering and history present in a fairly small area. SIXEP plant is one of two effluent treatment plants that manage discharges of radioactivity across the whole site. This is a highly complex engineered system. The plant works by capturing radioactivity present in a mobile form in the aqueous waste stream into an immobile solid form by a process of filtration and ion exchange. The SIXEP plant was commissioned in the mid 1980's and immediately resulted in the reduction of radioactivite discharge from the Sellafield site to less than $1 \%$ of the prior level. A schematic diagram of the SIXEP is shown in Fig.6.

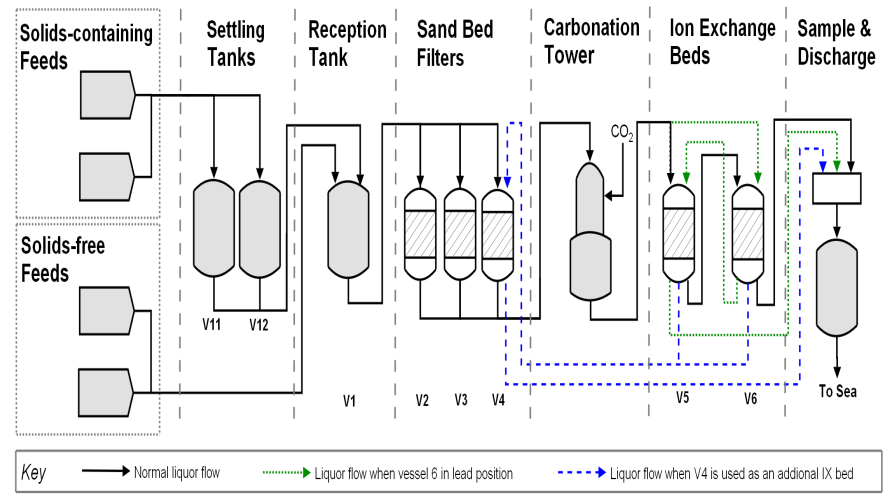

Figure 6: SIXEP Schematic [33] 
The feeds into the SIXEP contain particulate materials, and a number of soluble radioactive isotopes predominantly, Caesium-137 and Strontium90. These soluble radioactive species are removed from the liquid effluent using an ion exchange media loaded in 2 ion exchange beds which operates in series (one lead bed and one lag bed). The lead bed is replaced with fresh media when it is exhausted, and the bed that previously operated in the lag position is promoted to the lead position. The filtration and carbonation steps are present to protect the exchange beds and have a secondary benefit of removing actinides. In order to ensure the continued removal of these two key radioactive isotopes, the plant is routinely operated on the basis of feeds meeting a set of Conditions for Acceptance (CfA). These CfA define the feed envelope in terms of the acceptable concentrations of inactive species which affect the efficiency of the process. A model of the SIXEP plant have been constructed by the National Nuclear Laboratory, UK (see [33]) in the gPROMS modelling sofware developed by Process Systems Enterprise Ltd [34]. The model uses an equation-oriented architecture to represent equipment, chemistry, physics, operating procedures and other relationships, to whatever degree of accuracy is required. Whilst the SIXEP model is robust and accurate, it does not predict discharges precisely down to several significant figures. This makes this a really challenging test-case. However, the model is being used to test new feed compositions to provide assurance that the plant can continue to operate effectively, i.e. ensuring the discharges of Caesium-137 and Strontium-90 are kept within the required limits. Predictions made by this model are used in real-world application to underpin discharges for site data that is publicly available from the UK environmental 
agency [33].

\subsection{Uncertainties Affecting the SIXEP}

There is uncertainty of the future feeds composition arising from the Sellafield site, leading to variability in the activity levels of Caesium-137 and Strontium-90 and other soluble species that affect the removal of these isotopes. This variability can cause undesirable consequences to the environment (i.e. the discharges of the two afore-mentioned radionuclides exceeds their desired levels). Therefore, it is nessessary to include this uncertainty into studies when using the SIXEP model to assess the risk associated with the SIXEP model, and identify thoes model parameters that contribute significantly to this variability. It should be noted that the uncertainty considered to affect the plant feeds are aleatory (i.e. random) in nature [1]. The consideration of this type of uncertainty leads to defining of a state vector $\mathbf{x}$ of 18 state variables of the SIXEP model $\mathbf{x}=x_{n}: n=1,2, . ., 18$, which are assumed to be described by the probability distributions given in Table.7. The schematic of the SIXEP model is shown in Fig.7.

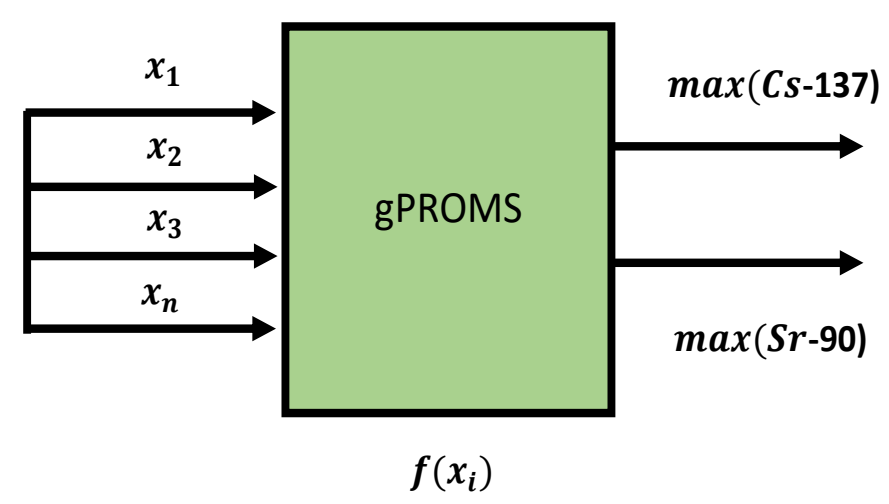

Figure 7: SIXEP Model Schematic 
Fig. 8 shows a deterministic simulation from the model using the set of mean values specified in Table.7. The results from the deterministic evaluation of the model shown in Fig.8 illustrates the activity levels of Caesium-137 and Strontium-90 with respect to time. It is to be noted that the simulation result shown in Fig.8, the SIXEP model has been started from a saved state representing steady state operation with the mean parameter values. From the figure (i.e. Fig.8), it can be see that there is a rise and drop in the activity levels of the Caesium-137 and Strontium-90 caused by the ion exchange bed change cycle. In this simulation, an ion exchange bed change occurs every 77 days (i.e. every 11 weeks). Hence, when a new ion exchange bed comes online, the activity discharges are low, and as the ion exchange media becomes saturated, activity breaks through the bed and thus produces a rising discharge profile which drops again following the next bed change (shown as the peaks in Fig.8). It should be noted that the scalar quantities of interest (i.e. performance variables) shown in Fig. 8 is the maximum activity of both radionuclides on the final day (616 $6^{\text {th }}$ day) of an ion exchange bed life, i.e.the number of days required to reach a new steady state. 
Table 7: SIXEP Model Input Parameters

\begin{tabular}{|l|l|l|l|l|}
\hline ParameterID & Mean & S.T.D & LowerBound & Upper Bound \\
\hline 1 & $0.50 \mathrm{E}+3$ & $0.66 \mathrm{E}+3$ & $0.01 \mathrm{E}+2$ & $6.63 \mathrm{E}+3$ \\
\hline 2 & $39.0 \mathrm{E}+3$ & $37.0 \mathrm{E}+3$ & $1.00 \mathrm{E}+3$ & $210 \mathrm{E}+3$ \\
\hline 3 & $1.05 \mathrm{E}+3$ & $359 \mathrm{E}+3$ & $0.11 \mathrm{E}+3$ & $3.00 \mathrm{E}+3$ \\
\hline 4 & $0.03 \mathrm{E}+3$ & $0.02 \mathrm{E}+3$ & $0.01 \mathrm{E}+4$ & $0.13 \mathrm{E}+4$ \\
\hline 5 & $46.0 \mathrm{E}-6$ & $36.0 \mathrm{E}-6$ & $3.00 \mathrm{E}-6$ & $494 \mathrm{E}-6$ \\
\hline 6 & $6.13 \mathrm{E}-3$ & $1.83 \mathrm{E}-3$ & $1.14 \mathrm{E}-3$ & $1.42 \mathrm{E}-3$ \\
\hline 7 & $1.59 \mathrm{E}-5$ & $1.28 \mathrm{E}-5$ & $0.25 \mathrm{E}-5$ & $14.7 \mathrm{E}-5$ \\
\hline 8 & $9.40 \mathrm{E}-6$ & $1.05 \mathrm{E}-5$ & $2.50 \mathrm{E}-7$ & $1.06 \mathrm{E}-4$ \\
\hline 9 & $15.9 \mathrm{E}+4$ & $7.10 \mathrm{E}+4$ & $1.90 \mathrm{E}-4$ & $4.81 \mathrm{E}+5$ \\
\hline 10 & $0.45 \mathrm{E}+2$ & $0.49 \mathrm{E}+2$ & $0.20 \mathrm{E}+1$ & $0.24 \mathrm{E}+3$ \\
\hline 11 & $2.00 \mathrm{E}+3$ & $0.62 \mathrm{E}+3$ & $0.73 \mathrm{E}+3$ & $4.00 \mathrm{E}+3$ \\
\hline 12 & $0.33 \mathrm{E}+2$ & $0.39 \mathrm{E}+2$ & $4.00 \mathrm{E}-2$ & $5.30 \mathrm{E}+2$ \\
\hline 13 & $0.14 \mathrm{E}+1$ & $0.30 \mathrm{E}+1$ & $3.00 \mathrm{E}-2$ & $0.37 \mathrm{E}+2$ \\
\hline 14 & $3.84 \mathrm{E}-6$ & $1.22 \mathrm{E}-5$ & $0.40 \mathrm{E}-12$ & $1.06 \mathrm{E}-4$ \\
\hline 15 & $3.50 \mathrm{E}-6$ & $2.82 \mathrm{E}-4$ & $2.74 \mathrm{E}-3$ & $4.61 \mathrm{E}-3$ \\
\hline 16 & $3.20 \mathrm{E}-6$ & $3.28 \mathrm{E}-6$ & $2.56 \mathrm{E}-7$ & $3.5 \mathrm{E}-5$ \\
\hline 17 & $2.38 \mathrm{E}-6$ & $2.93 \mathrm{E}-6$ & $2.50 \mathrm{E}-11$ & $2.50 \mathrm{E}-5$ \\
\hline 18 & $2.00 \mathrm{E}+6$ & $2.79 \mathrm{E}+5$ & $7.03 \mathrm{E}+5$ & $3.00 \mathrm{E}+6$ \\
\hline & & & & \\
\hline & & & \\
\hline & & &
\end{tabular}

Henceforth, the state variables $\mathbf{x}=x_{n}: n=1,2, . ., 18$ map out two performance variables defined by a vector $\mathbf{y}=y_{z}: z=1,2$. Propagating the 
uncertainties from the state variables via Monte Carlo simulation through the model gives rise to variability in the performance variables. The uncertainty propagation has been performed by generating 1000 samples and the maximum concentration of the two radionuclides are shown in the histograms given in Fig.9 and 10. The failure criteria $(F=X: g(X) \leq 0)$ of the model has been defined as when the performance variables exceeds unity.

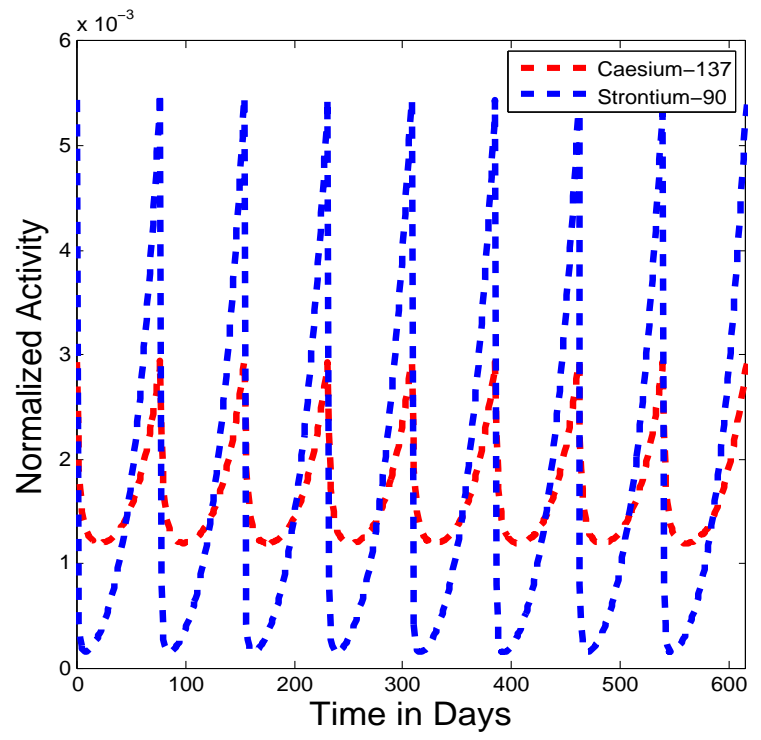

Figure 8: Normalised Deterministic Simulation from SIXEP Model 


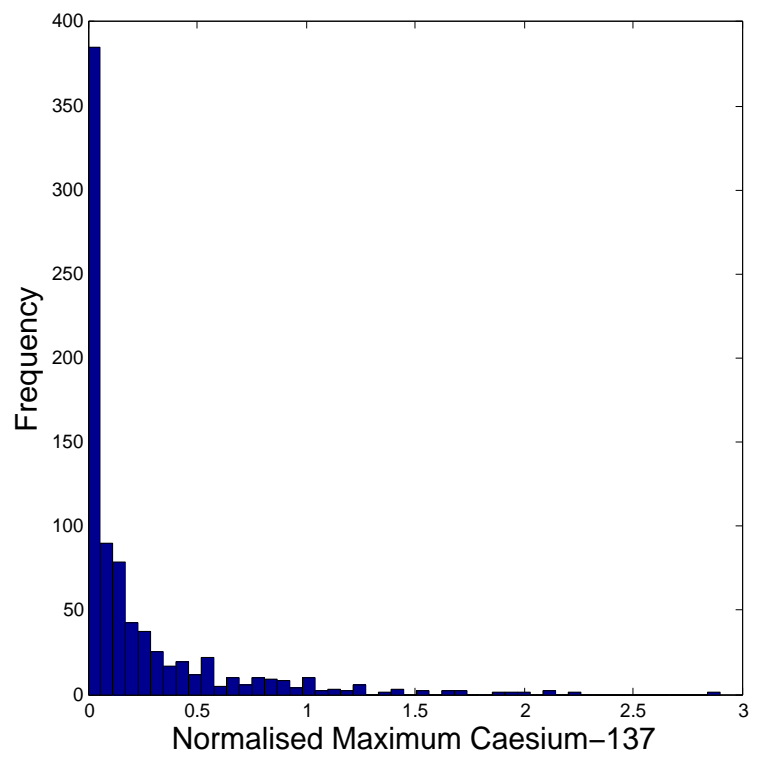

Figure 9: Normalized Variability in Maximum Caesium-137

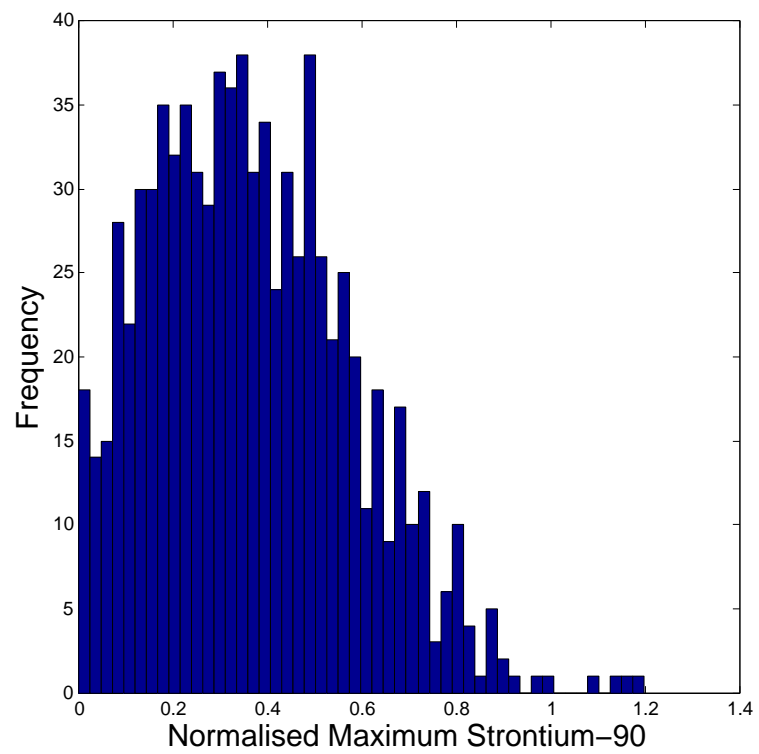

Figure 10: Normalized Variability in Maximum Strontium-90

Further, the reliability and sensitivity analysis technique discussed in this 
paper is been adopted to quantify the failure probability, and identify the contributions of the state variables to the variation in the performance variable.

\subsection{Construction of ANN for Reliability and Sensitivity Analysis}

As a single evaluation of the model requires approximately 1200 seconds for running a single process, ANN have been used as a substitute to speed up the time required for reliability and sensitivity analysis. To construct the network, training set $D_{\text {train }}(x, y)$ of sample size $N_{\text {train }}=1021$ have been obtained by evaluating the model repeatedly. Specifically, the training data set $D_{\text {train }}(x, y)$ have been generated via the LHS algorithm, considering the 18 state variables to be uniformly distributed. The idea behind using uniform distributions is to explore the admissible range of variability within each of the state variable. The hyper-parameters of the uniform distributions chosen to represent the state variables are identical to those specified in Table.7. Scalar quantities of the performance variables $\mathbf{y}=y_{1}, y_{2}$ have been computed, where $y_{1}$ and $y_{2}$ represents the maximum concentration of Caesium-137 and Strontium-90 respectively. The choice of the ANN architecture is vital for an accurate representation of the SIXEP model. In particular, three hidden layer configuration $(18,9,2)$ have been chosen.

\subsection{Training multiple Identical $A N N$}

Here, two sets $Z_{1}=N_{i}, i=1,2, \ldots M$ and $Z_{2}=N_{i}, i=1,2, \ldots M$ containing $M=1000$ ANNs have been trained. As done in the previous examples, $100 \%$ of the training samples have been used to train all the ANNs in $Z_{1}$, while $80 \%$ of the training samples have been used to train the ANNs in $Z_{2}$ 
and the remaining $20 \%$ used for validation. The posterior probability of the ANNs in $Z_{1}$ has been estimated by assigning uniform prior probability $P\left(N_{k}\right)=1 / M$ to each ANN. In addition, the $R^{2}$ validation errors have been computed for the ANNs in $Z_{2}$. For brevity, a summary of the top and bottom 5 ANNs based on their posterior probability and variance are shown in Tables 8-11. The same tables also show the $R^{2}$ validation error for a robust comparison.

Table 8: Top 5 ANN for Max Caesium-137

\begin{tabular}{|l|l|l|l|}
\hline$A N N$ & $\left.\sigma_{k}^{2}\left(Z_{1}\right)\right)$ & $P\left(D_{\text {train }} \mid N_{k}\right)\left(Z_{1}\right)$ & $R^{2}\left(Z_{2}\right)$ \\
\hline 1 & $0.18 \mathrm{E}-6$ & 0.162 & 0.9623 \\
\hline 2 & $0.19 \mathrm{E}-6$ & 0.116 & 0.9498 \\
\hline 3 & $0.20 \mathrm{E}-6$ & 0.107 & 0.9586 \\
\hline 4 & $0.21 \mathrm{E}-6$ & 0.058 & 0.9465 \\
\hline 5 & $0.22 \mathrm{E}-6$ & 0.046 & 0.9588 \\
\hline
\end{tabular}


Table 9: Bottom 5 ANN for Max Caesium-137

\begin{tabular}{|l|l|l|l|}
\hline$A N N$ & $\sigma_{k}^{2}\left(Z_{1}\right)$ & $P\left(D_{\text {train }} \mid N_{k}\right)\left(Z_{1}\right)$ & $R^{2}\left(Z_{2}\right)$ \\
\hline 1 & $1.62 \mathrm{E}-2$ & 0.0029 & 0.8839 \\
\hline 2 & $1.67 \mathrm{E}-2$ & 0.0047 & 0.8880 \\
\hline 3 & $1.72 \mathrm{E}-2$ & 0.0087 & 0.8990 \\
\hline 4 & $1.77 \mathrm{E}-2$ & 0.0066 & 0.6205 \\
\hline 5 & $1.83 \mathrm{E}-2$ & 0.0012 & 0.6140 \\
\hline
\end{tabular}

Table 10: Top 5 ANN for Max Strontium-90

\begin{tabular}{|l|l|l|l|}
\hline$A N N$ & $\sigma_{k}^{2}\left(Z_{1}\right)$ & $P\left(D_{\text {train }} \mid N_{k}\right)\left(Z_{1}\right)$ & $R^{2}\left(Z_{2}\right)$ \\
\hline 1 & $1.62 \mathrm{E}-6$ & 0.165 & 0.9275 \\
\hline 2 & $1.18 \mathrm{E}-6$ & 0.122 & 0.9330 \\
\hline 3 & $1.10 \mathrm{E}-6$ & 0.087 & 0.9594 \\
\hline 4 & $1.06 \mathrm{E}-6$ & 0.066 & 0.9588 \\
\hline 5 & $1.04 \mathrm{E}-6$ & 0.047 & 0.9517 \\
\hline
\end{tabular}


Table 11: Bottom 5 ANN for Max Strontium-90

\begin{tabular}{|l|l|l|l|}
\hline$A N N$ & $\sigma_{k}^{2}\left(Z_{1}\right)$ & $P\left(D_{\text {train }} \mid N_{k}\right)\left(Z_{1}\right)$ & $R^{2}\left(Z_{2}\right)$ \\
\hline 1 & $1.20 \mathrm{E}-2$ & 0.0016 & 0.8892 \\
\hline 2 & $1.18 \mathrm{E}-2$ & 0.0012 & 0.7948 \\
\hline 3 & $1.10 \mathrm{E}-2$ & 0.0008 & 0.8993 \\
\hline 4 & $1.06 \mathrm{E}-2$ & 0.0066 & 0.8969 \\
\hline 5 & $1.04 \mathrm{E}-2$ & 0.0047 & 0.8918 \\
\hline
\end{tabular}

Again, as shown in Tables 8-11, there is no correlation between the $R^{2}$ validation error and the posterior probability computed for each ANN (i.e. a high $R^{2}$ does not indicate the best model). For example, in Table. 8 when comparing the $4^{\text {th }}$ and $5^{\text {th }}$ ANNs, the $R^{2}$ value of the $5^{\text {th }}$ is 0.9588 which is greater than the $R^{2}$ value of the $4^{\text {th }}$ ANN (0.9456). However, the posterior probability of the $4^{\text {th }}$ ANN is greater than the posterior probability of the $5^{\text {th }}$ ANN. This confirms that a high $R^{2}$ validation error does not mean a better model.

\subsection{Robust Estimate of Failure Probability}

In this section, the ANNs in $Z_{1}$ have been adopted to implement the proposed approach in order to compute a robust estimate of the failure probability. Specifically, $10^{4}$ samples have been used to compute the failure probability $\hat{p_{F}}$ in each ANN. To reduce the computational time for this stage, parallelization strategy have been adopted to speed up the analysis (i.e. 20 parallel workers used). Then, the proposed approach has been used 
to compute the robust estimate of $\hat{p_{F}}$. Finally, confidence intervals of robust estimate quantifying the model uncertainties has been estimated based on Eqs. (12) and (13), and the result is shown in Fig.11.

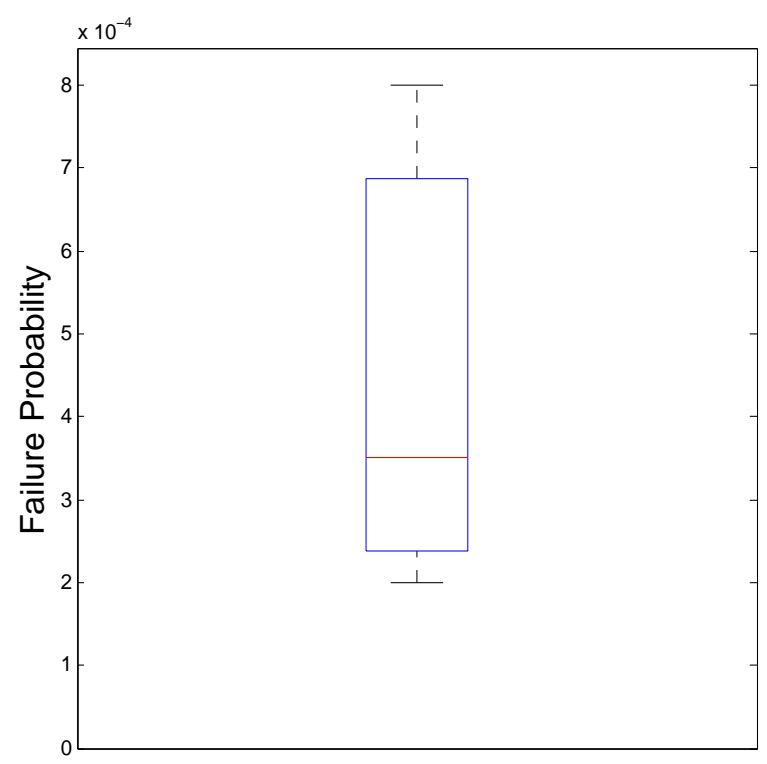

Figure 11: Confidence Intervals in Robust Prediction of the Probability of Failure

Although the confidence interval shown in Fig.11 is fairly wide, the most probable estimate of the true failure probability is represented by the mean of the interval(i.e. shown in red).

\subsection{Robust Estimate of Sensitivity Indices}

In this section, the ANNs in $Z_{1}$ have been adopted to implement the proposed approach in order to compute robust estimates of the sensitivity indices. Specifically, $10^{5}$ samples have been used to compute the sensitivity indices in each ANN adopting 20 workers in order to reduce the computational time. Then, the proposed approach has been used to compute a robust 
estimate of $S_{i}$ and $T_{i}$. Finally, the model uncertainty propagated to the robust estimates have been quantified in terms of confidence intervals (see Figs. $12-15)$.

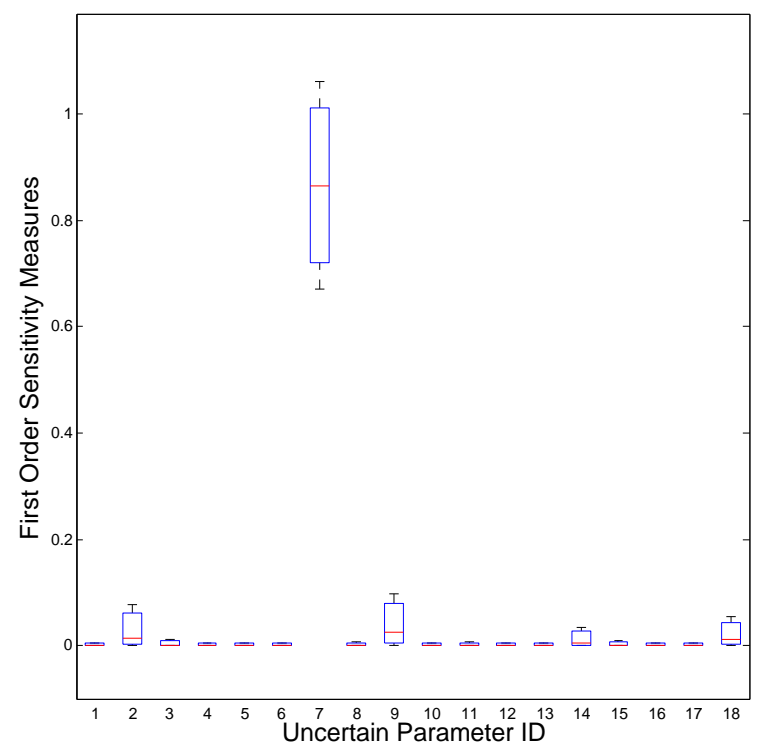

Figure 12: Confidence Intervals in Robust Prediction of First Order Sensitivity Indices (contributions to Cs-137) 


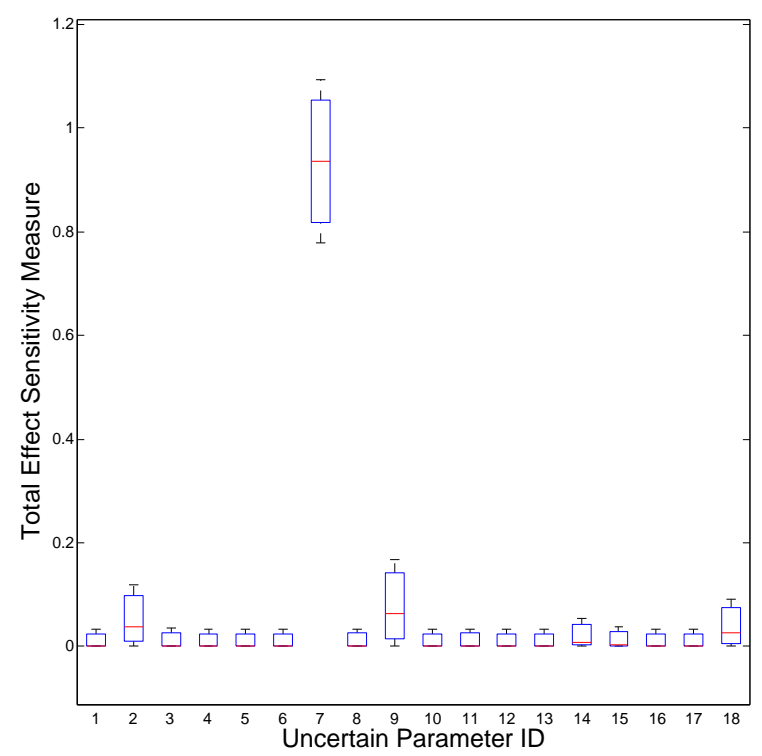

Figure 13: Confidence Intervals in Robust Prediction of Total Effect Sensitivity Indices (contributions to Cs-137)

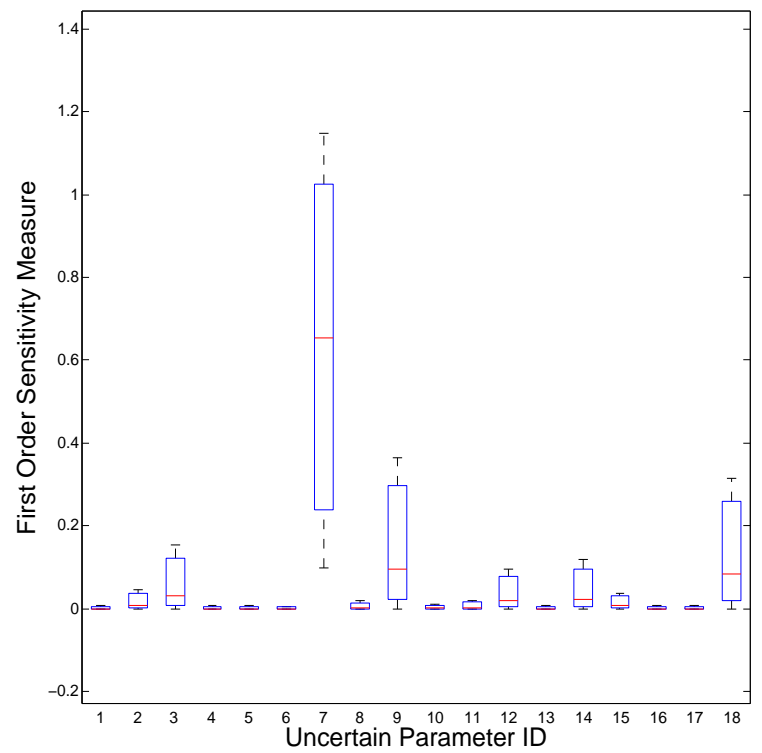

Figure 14: Confidence Intervals in Robust Prediction of First Order Sensitivity Indices (contributions to Sr-90) 


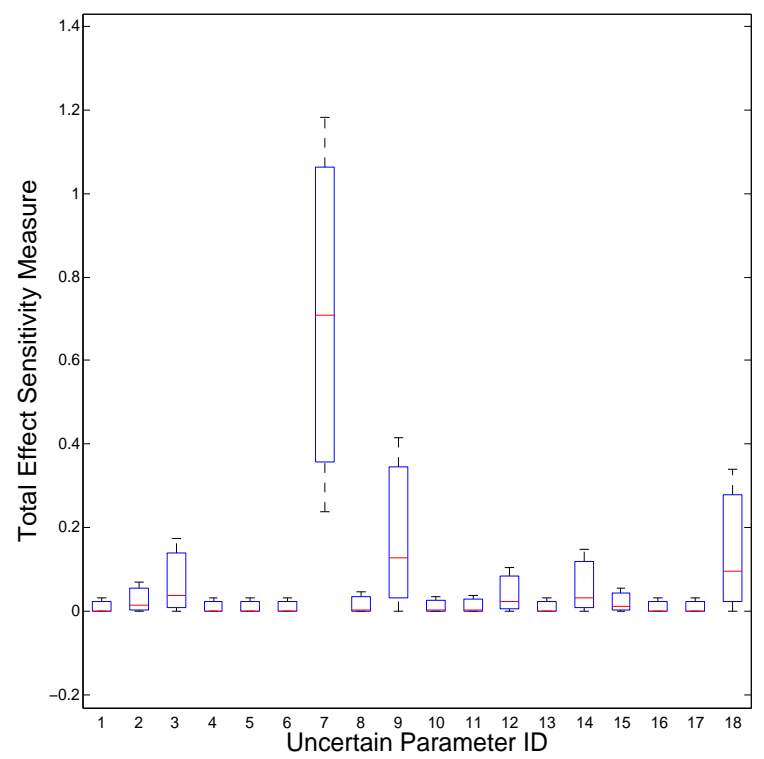

Figure 15: Confidence Intervals in Robust Prediction of Total Effect Sensitivity Indices (contributions to Sr-90)

From the results shown in Figs. 12-15, the state variables that have higher contributions (i.e. $7^{\text {th }}$ parameter) tend to have larger confidence intervals. A possible reason for these large intervals may be that the performance of an ANN reduces when estimating significant variables as a result of noise and other factors not known to the authors. However, the expected value (i.e. shown in red) is the most likely estimate that is to be taken as the true value when adopting the proposed approach.

\section{Conclusions}

Reliability and sensitivity analysis of complex models are computationally expensive due to the large number of model evaluations required to compute their robust measures. In this paper, an ANN is being used as substitutes 
for an expensive model to speed up the time required for the aforementioned analysis. However, the use of ANN for these kind of analysis introduces additional uncertainties into the predicted quantity. Therefore, it is vital to quantify the uncertainties in order to ensure a robust prediction. On the other hand, training a unique ANN architecture repeatedly results to variability in terms of their performance. This variability is as a result of the error function within each ANN being trapped in a different local minima. Hence, an additional uncertainty is introduced due to the lack of knowledge about the best performing ANN. Consequently, the use of cross-validation technique (i.e. $k-f o l d)$ to evaluate the performances of these ANNs based on their $R^{2}$ value is not an adequate measure due to the possibility to having a low $R^{2}$ value for a good ANN, and a high $R^{2}$ value for an ANN that does not fit the model adequately. In addition, the use of only part of the data set to train the ANN is wasteful of information, and drastically decreases accuracy in estimating the weight parameters of the ANN. Hence, we postulate that the use of cross-validation technique to select the best ANN out of a set of ANN with identical architecture introduces biassing and reduces the robustness of the predicted quantity.

Therefore, a novel approach has been presented to enhance the accuracy of the prediction (i.e. robustness) made by an ANN and quantify the model uncertainties in terms of confidence intervals. The proposed approach combines Bayesian model selection and model averaging technique into a unified framework. The applicability of the proposed approach is demonstrated in two numerical examples, and is further applied to a real case study concerning a radioactive waste management plant (SIXEP). The results obtained 
from this study shows that the true value of the quantity to be predicted by the ANN is close to the expected value given from our proposed approach, and within the confidence bounds that quantifies the model uncertainty. This model uncertainty quantification is of paramount importance in safety critical applications, in particular when few data representative are used to train an Artificial Neural Network.

\section{Acknowledgements}

This work has been partially supported by the EPSRC Grant EP/M018717/1 (Smart on-line monitoring for nuclear power plants (SMART)) and by the IRSES Marie Curie action of the European Union FP7-PEOPLE-2013-IRSES (Large Multipurpose Platforms for Exploiting Renewable Energy in Open Seas (PLENOSE)).

[1] J. C. Helton, D. E. Burmaster, Guest editorial: treatment of aleatory and epistemic uncertainty in performance assessments for complex systems, Reliability Engineering \& System Safety 54 (2) (1996) 91-94.

[2] E. Patelli, D. A. Alvarez, M. Broggi, M. de Angelis, Uncertainty management in multidisciplinary design of critical safety systems, Journal of Aerospace Information Systems 12 (2015) 140-169. doi:10.2514/1.I010273.

[3] P. Bjerager, On computation methods for structural reliability analysis, Structural Safety 9 (2) (1990) 79-96.

[4] E. Cashwell, C. Everett, Monte-Carlo methods, Pergamon, London, 1959. 
[5] R. Melchers, Importance sampling in structural systems, Structural safety 6 (1) (1989) 3-10.

[6] O. Ditlevsen, P. Bjerager, R. Olesen, A. Hasofer, Directional simulation in gaussian processes, Probabilistic Engineering Mechanics 3 (4) (1988) $207-217$.

[7] P. Koutsourelakis, H. Pradlwarter, G. Schuëller, Reliability of structures in high dimensions, part i: algorithms and applications, Probabilistic Engineering Mechanics 19 (4) (2004) 409-417.

[8] M. de Angelis, E. Patelli, M. Beer, Advanced line sampling for efficient robust reliability analysis, Structural Safety 52 (2015) 170-182.

[9] S.-K. Au, J. L. Beck, Estimation of small failure probabilities in high dimensions by subset simulation, Probabilistic Engineering Mechanics 16 (4) (2001) 263-277.

[10] S.-K. Au, E. Patelli, Rare event simulation in finite-infinite dimensional space, Reliability Engineering \& System Safety 148 (2016) 67-77.

[11] A. Saltelli, K. Chan, E. M. Scott, et al., Sensitivity analysis, Vol. 1, Wiley New York, 2000.

[12] T. Homma, A. Saltelli, Importance measures in global sensitivity analysis of nonlinear models, Reliability Engineering \& System Safety 52 (1) (1996) 1-17.

[13] E. Patelli, H. M. Panayirci, M. Broggi, B. Goller, P. Beaurepaire, H. J. Pradlwarter, G. I. Schuëller, General purpose software for efficient un- 
certainty management of large finite element models, Finite elements in analysis and design 51 (2012) 31-48.

[14] E. Patelli, COSSAN: A Multidisciplinary Software Suite for Uncertainty Quantification and Risk Management, Springer International Publishing, Cham, 2016, pp. 1-69.

[15] G. Baroni, S. Tarantola, A general probabilistic framework for uncertainty and global sensitivity analysis of deterministic models: A hydrological case study, Environmental Modelling \& Software 51 (2014) $26-34$.

[16] C. M. Bishop, Neural networks for pattern recognition, Oxford university press, 1995.

[17] J. Cheng, Q. Li, R.-c. Xiao, A new artificial neural network-based response surface method for structural reliability analysis, Probabilistic Engineering Mechanics 23 (1) (2008) 51-63.

[18] N. Pedroni, E. Zio, G. E. Apostolakis, Comparison of bootstrapped artificial neural networks and quadratic response surfaces for the estimation of the functional failure probability of a thermal-hydraulic passive system, Reliability Engineering \& System Safety 95 (4) (2010) 386-395.

[19] P. Secchi, E. Zio, F. Di Maio, Quantifying uncertainties in the estimation of safety parameters by using bootstrapped artificial neural networks, Annals of Nuclear Energy 35 (12) (2008) 2338-2350.

[20] F. Cadini, E. Zio, V. Kopustinskas, R. Urbonas, A model based on bootstrapped neural networks for computing the maximum fuel cladding 
temperature in an rmbk-1500 nuclear reactor accident, Nuclear Engineering and Design 238 (9) (2008) 2165-2172.

[21] D. E. Rumelhart, G. E. Hinton, R. J. Williams, Learning internal representations by error propagation, Tech. rep., DTIC Document (1985).

[22] T. Nilsen, T. Aven, Models and model uncertainty in the context of risk analysis, Reliability Engineering \& System Safety 79 (3) (2003) 309-317.

[23] G. B. Kingston, H. R. Maier, M. F. Lambert, Bayesian model selection applied to artificial neural networks used for water resources modeling, Water Resources Research 44 (4).

[24] L. Wasserman, Bayesian model selection and model averaging, Journal of mathematical psychology 44 (1) (2000) 92-107.

[25] E. Zio, A study of the bootstrap method for estimating the accuracy of artificial neural networks in predicting nuclear transient processes, IEEE Transactions on Nuclear Science 53 (3) (2006) 1460-1478.

[26] M. Bayarri, J. Berger, J. Cafeo, G. Garcia-Donato, F. Liu, J. Palomo, R. Parthasarathy, R. Paulo, J. Sacks, D. Walsh, Computer model validation with functional output, The Annals of Statistics (2007) 1874-1906.

[27] M. J. Bayarri, J. O. Berger, R. Paulo, J. Sacks, J. A. Cafeo, J. Cavendish, C.-H. Lin, J. Tu, A framework for validation of computer models, Technometrics.

[28] M. C. Kennedy, A. O'Hagan, Bayesian calibration of computer models, 
Journal of the Royal Statistical Society: Series B (Statistical Methodology) 63 (3) (2001) 425-464.

[29] D. G. Kleinbaum, M. Klein, Maximum likelihood techniques: An overview, in: Logistic regression, Springer, 2010, pp. 103-127.

[30] T. H. Wonnacott, R. J. Wonnacott, Introductory statistics, Vol. 19690, Wiley New York, 1972.

[31] J. C. Helton, F. J. Davis, Latin hypercube sampling and the propagation of uncertainty in analyses of complex systems, Reliability Engineering \& System Safety 81 (1) (2003) 23-69.

[32] A. Saltelli, Making best use of model evaluations to compute sensitivity indices, Computer Physics Communications 145 (2) (2002) 280-297.

[33] S. Owens, M. Higgins-Bos, M. Bankhead, J. Austin, Using chemical and process modelling to design, understand and improve an efluent treatment plant., NNL Science, 3, (2015) 4-13.

[34] U. G. gPROMS, Process system enterprise ltd, London, United Kingdom. 